\title{
A Model for Calculating ACCC Resistance Based on Fit Method
}

\author{
Beier Yuan', Xu-Dong Zhang ${ }^{2}$ \\ School of Energy and Power Engineering, Nanjing University of Science and Technology, No.200 \\ Xiaolingwei Street, Nanjing 210093, China \\ E-mail: beyuan47@163.com
}

Keywords: FACTS; HVDC; ACCC resistance; magnetic effect; fit method

Abstract. Aluminum Conductor Composite Core (ACCC) is expected as an important conductor in Flexible Alternative Current Transmission Systems (FACTS) and High Voltage Direct Current (HVDC), compared with Aluminum Conductor Steel-Reinforced (ACSR), has the characteristics of high strength, high temperature resistance and corrosion resistance. The method for calculating ACSR resistance should not be used to calculate ACCC resistance, since there is no magnetic effect in the ACCC. Due to the lack of theoretical research and related experiments on the calculation parameters of ACCC resistance, the application and development of ACCC have been greatly limited. To address this problem, a model of ACCC resistance is proposed based on fit method in this paper. In order to validate the proposed model, an experiment platform is set up and the experiment results are presented and discussed.

\section{Introduction}

ACCC combines the two major characteristics of energy saving and capacity expansion, and is a unique "green" conductor. Compared with ACSR, it has the characteristics of high strength, high temperature resistance and corrosion resistance [1-3]. As a transmission line carrier, it can effectively improve the capacity of transmission lines, and is expected to replace the traditional ACSR in both of FACTS and HVDC in the future [4-5].

However, due to the differences between the material and structure of ACCC and ACSR, the resistance parameters must be different. Consequently, the lack of modeling method of ACCC resistance at present leads to the development of ACCC limited. Therefore, based on that, the model for calculating ACCC resistance is proposed in this paper, and the estimated results have been validated by experiments after using fit method.

\section{Model for Calculating ACCC resistance}

\subsection{Differences in Composition Compared with ACSR}

There is a big difference in the inner conductor between ACCC and ACSR. The inner core of ACCC is cylindrical and carbon fiber composite one, while traditional ACSR is steel in it, which will lead to the changes of resistance and other parameters (Figure 1).

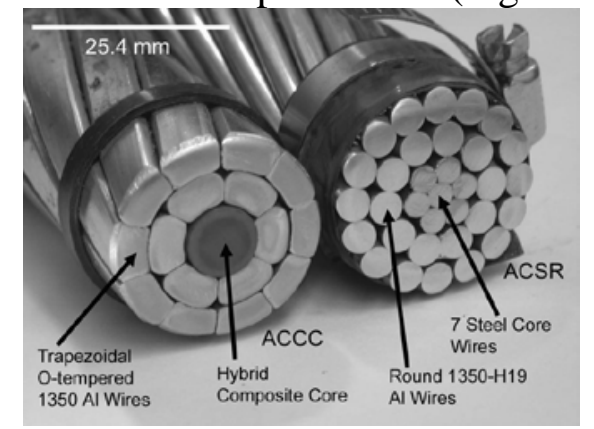

Figure 1 Section of domestic ACCC and ACSR [6]

Especially in the calculation of resistance, the ACCC carbon fiber composite core has no 
magnetic conductivity and electrical conductivity due to the covering of insulating resin. So there is no eddy current magnetic loss when the conductor is loaded current. Consequently, the resistance increment caused by the hysteresis vortex in the resistance of wire can be approximately neglected.

\subsection{Some Common Model for Calculating Resistance}

Here are some models for calculating ACSR resistance. Some scholars have proposed the influence of hysteresis and eddy current losses in the form of equivalent steel core permeability, and thus the electromagnetic parameters of the wire are described according to the electromagnetic theory. The electromagnetic model of ACSR resistance is constructed. According to [7, 8], each layer of wire resistance and inductance parameter are used for modeling respectively, and then the conductor impedance electromagnetic model is established. Taking a structure of the three layer of aluminum conductor as an example, the model can be shown in figure 2:

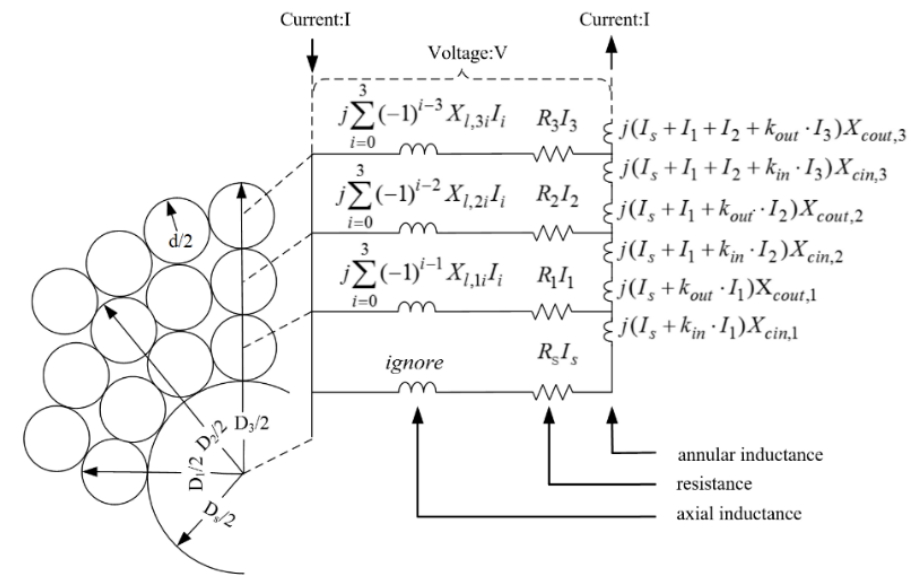

Figure 2 Electromagnetic model of wire impedance parameter

Formula (1) is expressed as the IEC standard model. $R\left(T_{c}\right)$ is the resistance when the conductor temperature is $T_{C}$ :

$$
R\left(T_{c}\right)=R_{r e f}\left(1+\alpha\left(T_{c}-T_{r e f}\right)\right)
$$

where: $R_{\text {ref }}$ is the resistance at reference temperature $T_{r e f}, T_{r e f}$ usually takes $20^{\circ} \mathrm{C}, \alpha$ is the temperature coefficient of resistance(TCR). In this model, in order to facilitate the practical engineering, the resistance is usually simplified as a linear function of conductor temperature, ignoring the influence of loading current on it.

Based on that, some scholars proposed a modified model for calculating the ACSR resistance as shown in formula (2):

$$
\left\{\begin{array}{l}
R(T, I)=\varphi(I) R_{r e f}\left[1+\alpha\left(T-T_{r e f}\right)\right] \\
\varphi(I)=\sum_{i=0}^{n} a_{i} I^{i}
\end{array}\right.
$$

where: $\varphi(I)$ is the current correction function for the resistance, represented as a polynomial function about the current, $\mathrm{n}$ is the polynomial order; $a_{i}$ is the polynomial coefficient which can reflect the nonlinear characteristics of the resistance with the changing of current; $1+\alpha\left(T-T_{\text {ref }}\right)$ is the temperature correction function for the resistance, same as the IEC standard model, which is a linear function of temperature.

According to 2.1 and above-mentioned theories, after taking the fact that ACCC has no magnetic effect into consideration, the IEC standard model is a suitable choice for ACCC resistance. Then $R_{\text {ref }}$ and $\alpha$ are both of parameters need to be fit by using least square method. 


\section{Experimental Verification and Result Analysis}

\subsection{Experimental Platform}

Figure 3 shows the experiment platform designed to solve the parameter calculations and verify their accuracy.

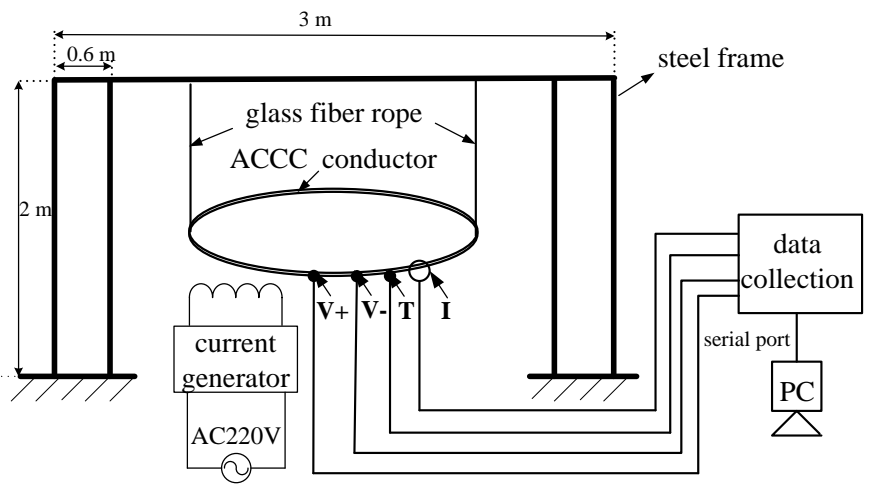

Figure 3 Schematic diagram of experiment platform for ACCC

The experimental platform is mainly composed of steel frame, experimental wire, strong current generator, temperature probe, differential voltage probe, current transformer, micro meteorological station, data acquisition device and PC machine. And glass fiber rope is used to hang ACCC for its thermo stability and low thermal conductivity. The ACCC used in the experiment is 6 meters long and the related parameters are shown in Table 1. And the high current generator is used to couple the experimental current to the wire. The micro meteorological station and the temperature probe are responsible for the measurement of meteorological phenomenon and the operating temperature of the conductor respectively. Importantly, differential voltage probe and current transformer are responsible for measuring the voltage and current waveform of the experimental section wire. After that, the data acquisition device is used to collect the above experimental data, and upload the data to the PC database.

By positioning the experiment platform in the closed laboratory, the condition can be seen as natural convection.

Table 1 Parameters of ACCC/TW-240/35

\begin{tabular}{|c|c|}
\hline Parameter name & Parameter value \\
\hline Specification & ACCC-240/35 \\
\hline Number of plies & 2 \\
\hline Number of aluminum strands & 25 \\
\hline Core diameter $/ \mathrm{mm}$ & 7.5 \\
\hline Outer diameter $/ \mathrm{mm}$ & 21.7 \\
\hline Nominal conductor cross-section $/ \mathrm{mm}^{2}$ & 287 \\
\hline Aluminum wire cross-section $/ \mathrm{mm}^{2}$ & 242.9 \\
\hline 20 degree DC resistance $/\left(\Omega \cdot \mathrm{km}^{-1}\right)$ & 0.1209 \\
\hline Allowable continuous temperature $/{ }^{\circ} \mathrm{C}$ & 160 \\
\hline
\end{tabular}

\subsection{Result and Analysis}

Figure 4 and 5 show the measured results of the ACCC resistance under different operating temperature and current conditions. 


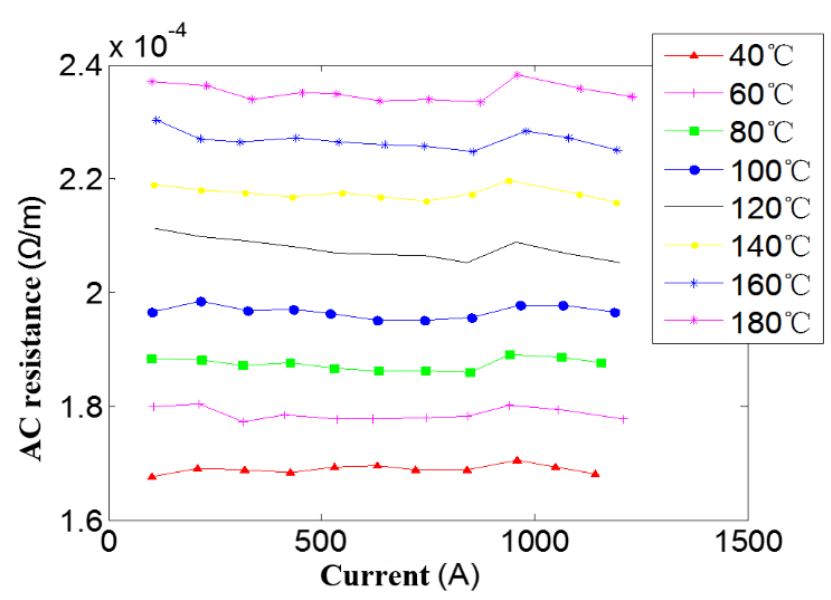

Figure 4 Experimental results of ACCC resistance

It's clearly that the resistance of the wire increases with the rise of the running temperature in figure 4. And it's shown that the value of ACCC resistance has nothing to do with the current changings apparently in figure 5.

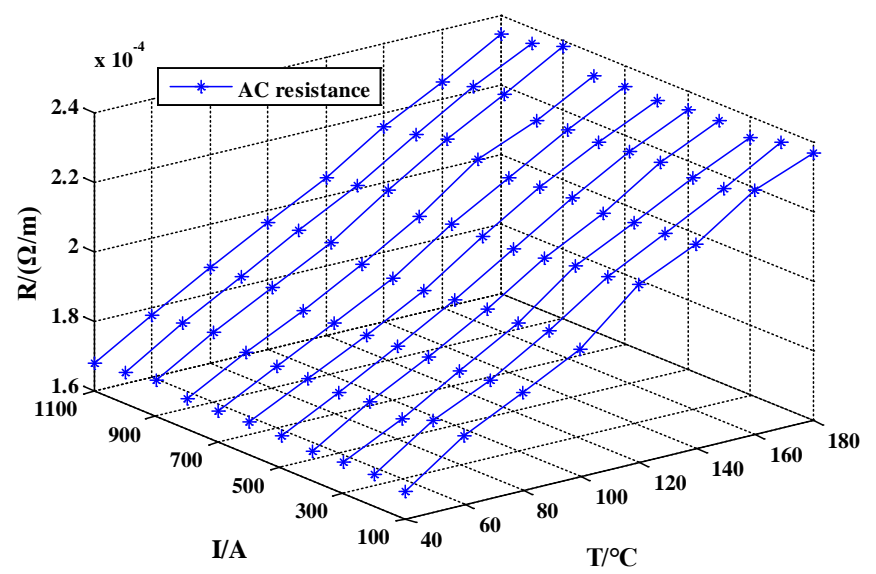

Figure 5 Experimental results of ACCC resistance in 3D

Figure 6 and 7 show that under the 500A current level the waveforms of voltage and current before and after filtering when the temperature of ACCC is $100^{\circ} \mathrm{C}$.

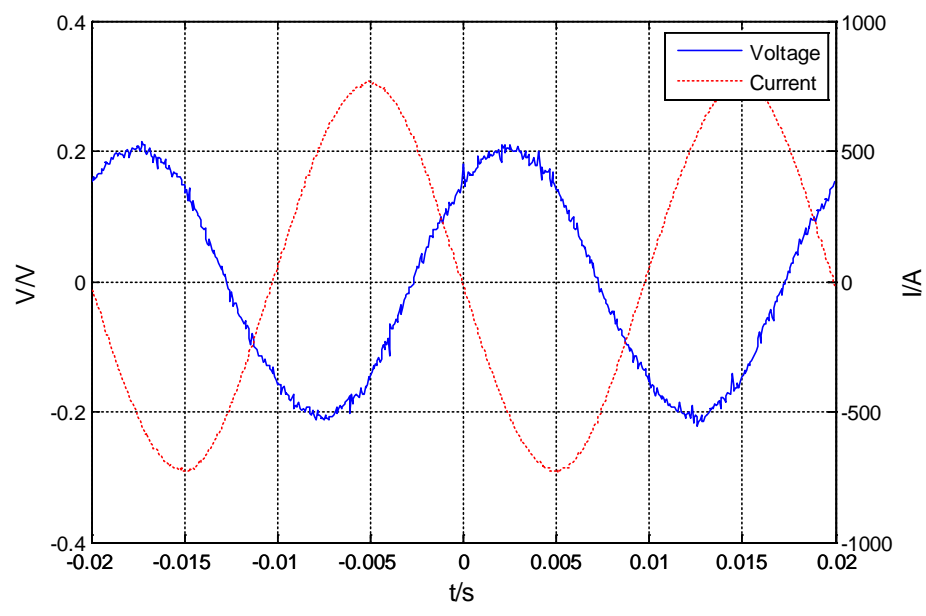

Figure 6 Waveforms of voltage and current before filtering 


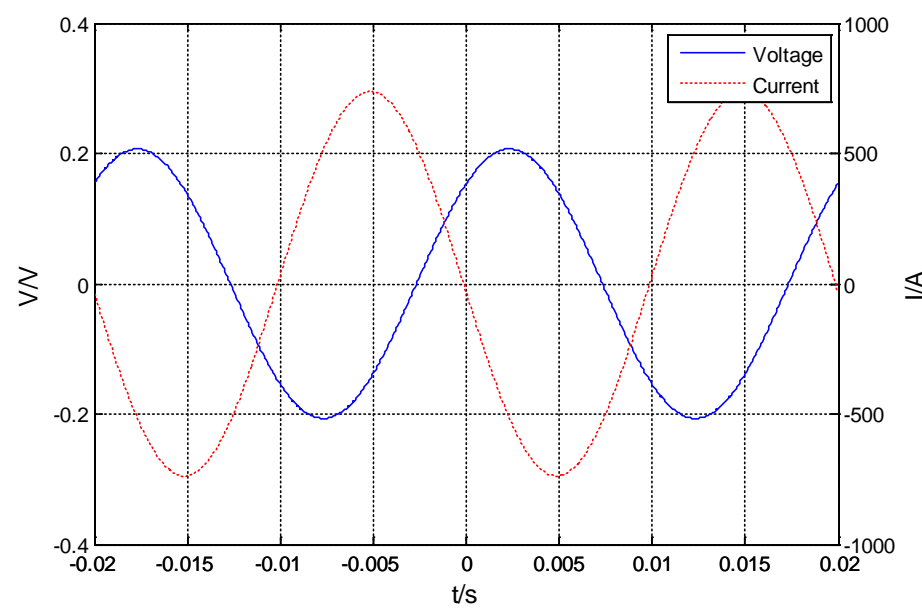

Figure 7 Waveforms of voltage and current after filtering

After obtaining the voltage and current waveforms of the experimental section, the measured value of the resistance is obtained by the vector current voltage method [9], as shown in figure 8 . The resistance of the wire which is estimated by using experimental data and fit method is also given in figure 8.

Table 2 Identification results of model parameters

\begin{tabular}{|c|c|}
\hline Resistance parameters & Estimated results \\
\hline$\alpha /{ }^{\circ} \mathrm{C}^{-1}$ & 0.0031 \\
\hline$R_{r e f} /\left(\Omega \cdot \mathrm{km}^{-1}\right)$ & 0.1595 \\
\hline
\end{tabular}

Table 2 gives the estimated values of the resistance parameters. As can be seen from the figure, the estimated values are relatively fit with the measured values, and the relative error is less than $1.2 \%$. So, it proves that the estimated values of resistance parameters are effective.

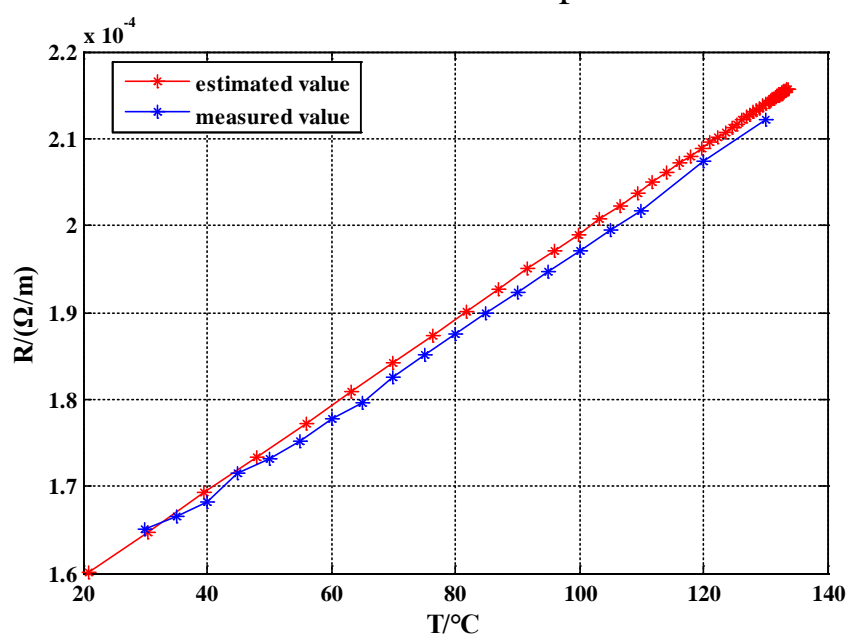

Figure 8 Estimated results and measured results of ACCC resistance

\section{Conclusion}

The model for calculating ACCC resistance based on fit method is proposed in this paper. And the experiment results have shown that least square method can estimate the parameters of ACCC resistance at different temperature effectively. Meanwhile, it also has proved that ACCC resistance changes without current. This, therefore, will provide theoretical basis for further research on 
ACCC.

\section{References}

[1] Burks B. Failure prediction analysis of an ACCC conductor subjected to thermal and mechanical stresses. Dielectrics and Electrical Insulation[J]. IEEE Transactions on Dielectrics and Electrical Insulation, 2010,17(2): 588-596.

[2] Alawar A, Bosze E J, Nutt S R. A composite core conductor for low sag at high temperatures[J]. IEEE Transactions on Power Delivery, 2005, 20(3):2193-2199.

[3] Vincent T. Morgan. Discussion of "High Temperature Ampacity Model for Overhead Conductors”[J]. IEEE Transactions On Power Delivery, 2003, 18(2):646-647.

[4] I. Zamora, A. J. Mazón, P. Eguía, R. Criado, C. Alonso, J. Iglesias, and J. R. Sáenz. High-temperature conductors: A solution in the uprating of overhead transmission lines[M]. In Proc. Power Tech, 2001, vol. 4.

[5] Deve H E, Clark R, Stovall J, et al. Field Testing of ACCR Conductor[J]. Electric Power Construction, 2007.

[6] Ahmad Alawar, Eric J. Bosze, Steven R. Nutt. A Composite Core Conductor for Low Sag at High Temperatures[J]. IEEE Transactions on Power Delivery, 2005, 20(3): 2193-2199.

[7] Dong Dai, Xiaohui Zhang, Juanjuan Wang. Calculation of AC Resistance for Stranded Single-Core Power Cable Conductors[J]. IEEE Transactions on Magnetics, 2014, 50(11): 1-4.

[8] J S Barrett, O Nigol, C J Fehervari, et al. A new model of AC resistance in ACSR conductors[J]. IEEE Transactions on Power Systems, 1986, 1(2): 198-208.

[9] Morgan V T, Findlay R D. The effect of frequency on the resistance and internal inductance of bare ACSR conduc-tors[J]. Journal of Bacteriology, 1991, 173(6):1902-10. 